Learnability of Kolmogorov-Easy Circuit Expressions Via Queries

\author{
José L. Balcázar \\ Harry Buhrman \\ Montserrat Hermo
}

Report LSI-95-8-R

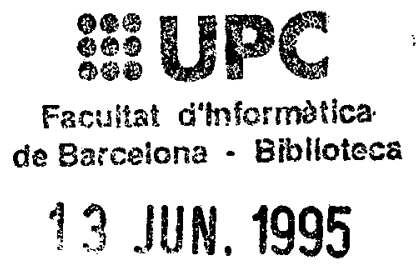




\title{
Learnability of Kolmogorov-Easy Circuit Expressions Via Queries
}

\author{
José L. Balcázar ${ }^{1}$, Harry Buhrman ${ }^{2}$, and Montserrat Hermo ${ }^{3}$
}

1 Dept. LSI, Edif. FIB, Universitat Politécnica de Catalunya, 08071 Barcelona, Spain

2 CWI, Kruislaan 413, Amsterdam, The Netherlands

3 Facultad de Informatica de San Sebastián, Universidad del País Vasco, Spain

\begin{abstract}
Circuit expressions were introduced to provide a natural link between Computational Learning and certain aspects of Structural Complexity. U.pper and lower bounds on the learnability of circuit expressions are known. We study here the case in which the circuit expressions are of low (time-bounded) Kolmogorov complexity. We show that these are polynomial-time learnable from membership queries in. the presence of an NP oracle. We also exactly characterize, in terms of advice classes, the sets that have such easy circuit expressions, obtain consequences regarding their lowness, and precisely identify the subclass whose circuit expressions can be learned from membership queries alone, by means of doubly tally polynomial time degrees. The extension of the results to various Kolmogorov complexity bounds is discussed.*
\end{abstract}

\section{Introduction}

This paper studies the learnability of circuit expressions in the "learning via -queries" model of Computational Learning. In this model, the learning algorithms interact with their environment, trying to grasp. a concept. The concept will be formally modeled as a set of encodings, which are themselves simply binary strings. The interaction is formalized by "queries", and these in turn are of the kind of questions suggested by the set-theoretic modeling of concepts. For instance, the simplest one is the "membership" query, in which the learner presents a binary string and asks for its classification as "in" or "out" the concept set; and the "subset" query presents (a finite encoding of) a set of words and asks whether that set is a subset of the concept set. We focus on the "bounded learning" model from [13], where it is not necessary to completely identify the concept: a length bound is given initially to the learner, and it must identify the concept up to that bound.

Concepts are usually represented in some manner. We study here the.representation: via circuit expressions. These are defined inductively just as regular

* These results were announced at EuroColt'95, Barcelona. Work partially supported by the EC through the Esprit program (project 7141, ALCOM-II, and Working Group 8556, NeuroColt), and through the HCM program (project CHRX-CT93-0451, COLORET Network), by the Ducth NWO, and by the Spanish DGICYT (project PB92-07099).E-mail: balqui@1si.upc.es, buhrman@cwi.nl, jiphehum@si.ehu.es 
expressions, by the operations of union, concatenation, and Kleene star, with the difference that boolean circuits are considered also as circuit expressions. We can therefore take unions of circuits, or Kleene stars of circuits. Their meaning is defined in the standard way (but see below for a complete description).

Circuit expressions were introduced in [14] to provide a natural link between Computational Learning and certain aspects of Structural Complexity. They share most interesting properties of the boolean circuit model, but overcome the inconvenience that a fixed boolean circuit has a fixed number of inputs and, therefore, accepts a subset of $\{0,1\}^{n}$ for some fixed $n$; circuit expressions may even accept infinite sets by using the Kleene star and union. In [14], learnability issues for this and other representation classes were related to the "representation finding" problem of Structural Complexity, and this allowed for a precise definition of the "computational power" of a learning protocol, given in terms of (relativizations of) the polynomial-time hierarchy.

For instance, following the intuitions there, it can be seen that it is possible to learn circuit expressions for a concept $A$ via membership queries if and only if $A$ is polynomial-time T-equivalent to a tally set. (This is argued in some more detail below.) The polynomial-time tally T-degrees, which were known to correspond to the so-called "self-producible circuits", characterize therefore the concepts for which:a specific learnability problem is solvable. This paper will pursue further this sort of connections.

Upper and lower bounds on the learnability of boolean circuits are known, and most of them carry over to circuit expressions. Actually, in the work made up to now, the distinction between circuits and circuit expressions is hardly worth to be made, being at most a small technical detail. Research in Structural Complexity shows that circuits for a set can be obtained from an oracle in $\Sigma_{2}^{p}$ plus an NP oracle relative to the set itself [10]. It is also known that it is possible to learn deterministically boolean circuits, from equivalence queries plus

- a $\Sigma_{3}^{p}$ oracle, or just with an NP oracle by a randomized algorithm [7]. Although there is motivation for the use of equivalence queries (e.g. connections to the "mistake-bounded" model), these fall into the category of "expensive" query types, in that for many applications it makes little sense to expect a teacher to answer equivalence queries.

However, for reasonably simple queries like membership, it is not difficult to see that circuits cannot be identified with polynomially many queries, regardless of the amount of computational power available [1]. As for other queries, in [14] the computing power needed to learn a "repetitive" variant of circuits is exactly . characterized by classes in the relativized polynomial time hierarchy. The survey [8] gives a precise account of many such results and related ones.

We study here the case in which the circuit expressions are of low timebounded Kolmogorov, complexity; specifically, the case in which they have logarithmically long descriptions, from which the (polynomial size) expressions can be recovered in polynomial time. There are two reasons. First, in many natural cases, this would be satisfactory enough, since frequently large circuits are built of replications of small ones; for instance, the quadratic circuit simulation of a 
time-bounded Turing machine is a very regular circuit consisting of a repeated fized-size pattern, and its Kolmogerov complexity is precisely logarithmic. Second, it is known from other approaches to Computational Learning (such as PAC) that sometimes concepts tliat are not (or not known to be) learnable in general become learnable under such "simplicity" conditions [11]. This paper proves that this is also the case with learning circuit expressions via queries.

First, we exactly characterize the sets that have such logarithmically easy circuit expressions, in order to know exactly what concepts are we fighting with. The proof is not immediate, but the difficulties can be solved by using techniques developed previously by the authors.

To analyze the learnability of these concepts, we point out some easy observations proving that they are PAC-learnable, and then we study the learnability from queries. We start from a naive prefix-search algorithm that uses, in a standard way, a "relativized NP" oracle, similar to the ones used in [14] for the general case. Then we show how to use membership queries to "un-relativize" the NP oracle. This means that we prove that for every set having logarithmically easy circuit expressions, these can be found with membership queries in the presence of an NP oracle, in polynomial time.

An interesting observation is that the analysis and the replacements of the oracles bear also consequences for the Structural Complexity of the classes studied, yielding so-called "lowness". properties for all such sets. Also, it is worth pointing out that our algorithms work by directly obtaining a logarithmically long seed that will produce, in polynomial time, a circuit expression for the concept.

A natural subclass to try to understand is that of those concepts for which the learnability can be performed using simply the most inexpensive queries, those of "membership", without resorting to additional oracles. We study this subclass and characterize it in terms of polynomial time degrees: for a concept $A$, circuit expressions can be learned from membership queries alone if and only if $A$ belongs to a polynomial-time doubly tally $T$-degree. (see the preliminaries for a definition). This class was already known, and characterizations of it, with an analysis of its inner structure, appear in [9].

The extension of the results to various Kolmogorov complexity bounds is also discussed. We prove that many of the technical properties on which our results are based also hold for other bounds, in particular for the polylog case. We extend some of the characterizations to these bounds. An interesting fact is that the corresponding conditions on the tally sets are no longer qualitative but quantitative: from $\log ^{2} n$ onwards, the tally sets must be defined by density conditions, while for the $\log n$ case we had to request a specific pattern of the words in the corresponding tally sets. The deep reason of this divergence is not fully understood. 


\section{Preliminaries}

\section{Complexity Theory}

Our notions and notations of Complexity Theory are standard; see [3]. Our sets consist of words over a single fixed alphabet with at least two symbols; specifically, we assume that the alphabet is $\{0,1\}$, to operate on words by means of boolean models of computation. The most basic of them are assumed known. For a set $A, A^{=n}$ is the set of words of length $n$ in $A$, and $A \leq n$ is the set of words of length up to $n$ in $A$.

We denote by $\langle.,$.$\rangle any easily computable, easily invertible pairing function,$ encoding a pair of words into a single one; we also assume it length-preserving, in the sense that if the arguments are replaced by others of the same length, the total length of the encoded pair is also the same.

Sets of sets are usually called classes. The class $\mathrm{P}$ consists of the problems solvable deterministically in polynomial time. If the polynomial time computation has access to an oracle set $A$, the resulting class is denoted $\mathrm{P}^{A}$. When $B \in \mathrm{P}^{A}$, we say that $B$ is polynomial time Turing reducible to $A$; if both are each reducible to the other, then we say that they are polynomial time Turing equivalent. For $C$ a complexity class, $C^{A}$ denotes the class where each machine in the class has access to the oracle $A$. The class of all sets polynomial time Turing equivalent to $A$ is denoted $E_{T}(A)$; this class is the (polynomial time Turing) degree of $A$. We use similarly a whole class of sets instead of a single set $A$ : so, $E_{T}(C)$ is the class of sets polynomial time Turing equivalent to some set in the class $C$.

We denote by SAT any NP-complete problem such as the well-known "Satisfiability" problem of boolean formulas. A set $S$ is sparse if the cardinality of $S \leq n$ is bounded by some polynomial. A set $T$ is tally if $T \subseteq\{0\}^{*}$. A set is doubly tally if it is tally and all of its words have length a power of 2 . The class of all doubly tally sets is denoted by Tally2.

Oracles are one way of providing computational devices with additional informátion. Advice words are another, and give rise to nonuniform complexity classes. The nonuniform class Full-P/log consists of all sets for which there exist advice words $w_{n}$ with $\left|w_{n}\right| \leq c \log (n)$ for some constant $c$, and a set $B \in \mathrm{P}$, such that for all $n$ it holds:

$$
\forall x(|x| \leq n),\left(x \in A \Longleftrightarrow\left\langle x, w_{n}\right\rangle \in B\right)
$$

Other advice classes are obtained using other families of bounds for the length of the advice words. For instance, using polynomials, we get $\mathrm{P} /$ poly, which is known to coincide with the problems that can be solved by having polynomial size circuits. An equivalent definition is $\bigcup_{S}$ is sparse $\mathrm{P}(S)$. A similar, less known characterization of Full-P/log is as follows:

Theorem 1. [5] Full- $\mathrm{P} / \log =\mathrm{P}($ Tally2)

Circuit expressions were introduced in [14]. They are constructed exactly as regular expressions, with the additional proviso that boolean circuits are circuit 
expressions. Hence, a circuit expression is a boolean circuit with a single output, or the sum of two circuit expressions, or a star operator applied to a circuit expression. The language denoted by a circuit expression is defined in the usual way: if the expression is a standard circuit, it denotes the set of words for which the output is 1; the sum corresponds to the union, and the star to the stardard Kleene star operator of regular languages. Circuit expressions can be evaluated in polynomial time [14].

We denote by $C E X(A, n)$ the set of circuit expressions $C$ such that $L(C)$ coincides exactly with $A$ up to size $n$.

\section{Bounded Learning}

Following [13], we model concepts as sets of words. We follow the "bounded learning" framework from that reference. The learner is a (usually polynomialtime) algorithm, sometimes with access to some oracle set. It is initially provided with a bound $n$ on the size of the representation of the target concept, and with a length bound $m$; its goal is to output a representation, of size no.larger than $n$, that correctly represents the target concept up to size $m$. Information about the target concept is obtained from a "teacher" able to somehow compute the answer to some selected set-theoretic questions. Basic queries are Membership. ("Is this word a member of the target set?") and Equivalence ("Does this representation correctly represent the target set up to the length bound?"); less frequent queries are Subset and Superset. Sometimes counterexamples are required for some of these queries, but in this paper we will not use this brand. See [13] for the exact notion of representation class and for precise formalizations of all these concepts.

We say that a class of sets is (Mem)-CEX-learnable if there is a (single) learning algorithm that learns circuit expressions for all sets in the class, using membership queries, in polynomial time. Sometimes an oracle (e.g. SAT) is assumed to be available, and we will explicitly indicate this fact:

Obviously a polynomial time learner only can write down as output a circuit expression of polynomial size, so that each CEX-learnable concept is in $\mathrm{P} / \mathrm{poly}$. The following is also immediate:

Proposition 2. If $\mathcal{C}_{1} \subseteq \mathcal{C}_{2}$ and $\mathcal{C}_{2}$ is (Mem)-CEX-learnable; then $\mathcal{C}_{1}$ is (Mem)CEX-learnable.

The connection with the theory of polynomial time degrees comes from the following fact, pointed out by Osamu Watanabe (personal communication). Results in the same spirit (for more general representation classes) appear in [14].

Proposition 3. If $\mathcal{C}$ is (Mem)-CEX-learnable then every concept in $\mathcal{C}$ is polynomial-time T-equivalent to some tally set.

The proof follows the guidelines of the characterization of the polynomialtime tally T-degrees by means of "self-producible circuits"; see for instance [3]. We simply sketch it here. Essentially, given a learnable set $A$, a tally set can 
encode in a standard way the circuit expression that the learning algorithm finds. Given the set as oracle, the tally set can be decided by running the learner and checking that the encoding is correct;-conversely, given the tally set as oracle, the corresponding circuit expression can be retrieved and evaluated.

On the other hand, if $A$ is T-equivalent to a tally set $T$ in polynomial time, a learning algorithm on input $n$ scans all of $0^{*}$ up to some polynomial $p(n)$ and, for each word, uses queries to $A$ to decide membership to $T$. Once a large enough initial segment of $T$ is known, it is easy to design a polynomial size circuit expression that simulates the machine reducing $A$ to $T$ on inputs of length up to $n$, which has built-in enough information about $T$ to compute $A$, up to size $n$, without further aid.

\section{Learning easy circuit expressions}

In order to give a precise definition of the subclass of circuit expressions we study, we introduce the resource-bounded Kolmogorov complexity classes. (See [12] for undefined notions and properties.) Fix any universal Turing machine $U$.' Define the sets of bounded Kolmogorov complexity strings. $K[f(n), g(n)]$ as follows:

Definition 4. $x \in \mathrm{K}[f(n), g(n)]$ if there exists $y,|y| \leq f(|x|)$, such that $U(y)=x$ in at most $g(|x|)$ steps.

Such $y$ is sometimes called a "seed" for $x$. We focus here in the class $\mathrm{K}[\log$,poly] in which functions from $O(\log n)$ are selected for $f$, and functions from $n^{O(1)}$ are selected for $g$. The chosen constants may depend on the set to be learned, but not, of course, on the length for which the circuit expression is desired.

Definition 5. A set $A$ has easy circuit expressions if and only if for some constant $c$, and for each $n$, there is $C_{n} \in C E X(A, n)$, of size $\left|C_{n}\right| \leq n^{c}$, such that $C_{n} \in \mathrm{K}\left[c \log n, n^{c}\right]$.

Before moving into learning such easy circuit expressions, it is worth to know something about the sets having easy circuit expressions. We have:

Theorem 6. A set $A$ can be decided by a family of easy circuit expressions if and only if $A \in$ Full-P/log.

Proof. Suppose first that $A \in$ Full-P/log: there exists a sequence of advice words $w_{n}$ with $\left|w_{n}\right| \leq c \log (n)$ for some constant $c$, and a set $B \in \mathrm{P}$, such that for all $n$ it holds:

$$
\forall x(|x| \leq n)\left(x \in A \Longleftrightarrow\left\langle x, w_{n}\right\rangle \in \dot{B}\right)
$$

Using standard techniques, it is easy to construct from $n$ and $w_{n}$ a polynomial size circuit $C_{j} \in \mathrm{K}\left[\log\right.$, poly] that recognizes each $A^{=j}$ for $j \leq n$. Each includes 
a simulating circuit for the polynomial time machine that computes the pairing function, having as "hardwired" (i.e. fixed to constants) inputs the advice bits of $w_{n}$ (not $w_{j}$ ) to pair it with its input, and then piped into a simulating circuit for the polynomial time machine accepting $B$ at length $\left|\left\langle 0^{j}, w_{n}\right\rangle\right|$; recall here that $($,$\rangle is assumed to be length-preserving. These circuits have polynomial size$ but simply consist of replications of constant-size subcircuits that apply the transition table of the machines, so they are easily described by their width and depth, plus $w_{n}$, with in total logarithmically many bits. They can be put together into an easy circuit expression for $A \leq n$, by means of the union operation. It is important to notice here that the logarithmically many constant gates encoding the advice word are the same for all the circuits in the circuit expression.

Suppose now that $A$ has easy circuit expressions; thus there exists $p(n)$ such that for all $n$ there exists a circuit expression $C E_{n}$ in such a way that $C E_{n} \in C E X(A, n),\left|C E_{n}\right| \leq p(n)$, and $C E_{n} \in \mathrm{K}[\log$, poly]. As a first approximation, consider the set $B \in \mathrm{P}$ formed by pairs $\langle x, s\rangle$ where $x$ is in the language recognized by the circuit expression produced by $U$ from $s$. The running time of $U$ is only allowed to be an appropriate polynomial.

Here is where the proof becomes nontrivial. It is easy to see that it is not enough to take simply the seeds as advice words. If, together with $x$, we give a seed of roughly the same length, it takes exponential time to find and evaluate an exceedingly large circuit expression; and, if we forbid this possibility, we are not fulfilling the definition of Full-P/log. The advice words to be taken are, instead, concatenations of the seeds for circuit expressions $C_{n}$ for $n$ a double power of 2 . This technique was introduced in [5], and a similar one is used below in theorems 10 and 11.

More precisely, each advice word will contain several seeds, corresponding to some selected lengths, and avoiding to store all of them. We will encode all the seeds for lengths $n=2^{2^{m}}$ and skip all the intermediate ones. Let $z_{m}$ be the concatenation of all the words $s_{2^{11}}, s_{2^{2} 2^{2}}, \ldots s_{2^{2 m}}$, separated with some markers or using a self-delimiting encoding to be able to retrieve each of them, and denote $m_{n}$ the natural fulfilling $2^{2^{m_{n}-1}}<n \leq 2^{2^{m_{n}}}$. Then the advice word for length $n$ will be $z_{m_{n}}$ : Easy computation shows that the length of $z_{m_{n}}$ is again logarithmic in $n$.

We can define the set $B$ as consisting of pairs. For each $x$, it holds $\langle x, s\rangle \in B$, where $s$ is now a concatenation of words, exactly if the $m_{|x|}$-th of them, when fed to $U$, yields in polynomial (in $|x|$ ) time a circuit expression accepting $x$. It is immediate that $B \in \mathrm{P}$ and that $x \in A \Longleftrightarrow\left\langle x, z_{m_{n}}\right\rangle \in B$ for $|x| \leq n$.

This last proof being constructive, it also indicates that in order to learn circuit expressions it is enough to find a way of computing the appropriate advice words. These, together with the length up to which the circuit expression is desired and a constant size program, are enough to fully reconstruct the desired circuit expression.

We move now to discuss learnability. Fix $A \in$ Full-P/log: From the fact that only polynomially many descriptions exist for easy circuit expressions, we obtain immediately an algorithm based on equivalence queries: simply, reconstruct all 
potential circuit expressions by cycling over all logarithmically long seeds, and ask each one as an equivalence query (which does not need here a counterexample). This obvious algorithm proves not only that equivalence queries suffice, but also, via a now standard transformation [1], that easy circuit expressions are PAC-learnable.

A serious objection is that allowing a number of equivalence queries similar to the number of potential hypothesis does not seem reasonable. We next allow ourselves the use of a set in $\mathrm{NP}^{A}$ to get rid of them, in two different forms. First; it is easy to see that the equivalence query can be answered by an oracle in $\mathrm{NP}^{A}$. Now it is not difficult to reduce the number of queries to a different set in NPA .

Theorem 7. Easy circuit expressions for every set $A \in$ Full-P/log can be learned in polynomial time, making logarithmically many queries to $\mathrm{NP}^{A}$.

Proof. Suppose $A \in$ Full-P $/ \log$. We will show how to construct the advice words, and then appeal to the constructive character of the previous characterization. We will say that the word $y$, with $|y| \leq c \log n$, is a "good advice" if $\forall u(|u| \leq n)$ $(\langle u, y\rangle \in B . \Longleftrightarrow u \in A)$. This predicate is in co-NP ${ }^{A}$. Let $G A$ be the following oracle set:

$$
G A=\left\{\left\langle z, 0^{n}\right\rangle|| z \mid \leq c \log n \wedge \exists y z \sqsubseteq y(|y|=c \log n \wedge y \text { is a "good advice") }\}\right.
$$

The set $G A$ belongs to co-NPA $\mathrm{N}^{A}$, and allows for a prefix-search procedure to calculate a good advice by asking $G A$ about consecutive prefixes. Thus, if $A \in$ Full-P/log then circuit expressions for $A$ can be learned in polynomial time, making logarithmically many queries to $\mathrm{NP}^{A}$.

Our interest centers now, therefore, on learning via membership queries to the target concept. We will prove that it is not necessary that the NP oracle is itself relativized to the target, by showing an algorithm that learns by querying separately the target and an unrelativized NP oracle.

Theorem 8. Let $A$ have easy circuit expressions, i.e. $A \in \mathrm{Full}-\mathrm{P} / \log$. Then, in the presence of an additional NP oracle, such easy circuit expressions for $A$ can be learned in polynomial time from membership queries.

Proof. Let $A$ be a set in Full-P/log, and let $n$ be the length bound up to which a circuit expression for $A$ must be correct as in the definition of bounded learning. For each word $w$ of $\operatorname{logarithmic~length,~say~} c \log n$, let $C_{w}$ be the circuit expression obtained using $w$ as seed for a fixed universal Turing machine $U$ during an appropriate polynomial number of steps, say $n^{c}$, as given by the definition of easy circuit expressions for $A$. By convention, if on input $u$ the universal machine does not output a valid circuit expression in the indicated time, we let $C_{w}$ denote a trivial circuit expression rejecting everything.

The kernel of the algorithm is a subprogram that, given two easy circuit expressions $C_{u}$ and $C_{w}$, tries to find a word on which they give different answers; this is where the NP oracle is used. If two circuit expressions give always the 
same answers, either both are correct of both are wrong, so it is unnecessary to keep both; one is discarded. Otherwise, the learner makes a membership query about the distinguishing word; the circuit expression that is inconsistent with the answer gets discarded. Only the correct one, that exists by hypothesis (and is unique after discarding those that give the same answers) will emerge out of the game.

The subprogram that uses an NP oracle to find a word that distinguishes two advice words followis a standard prefix search procedure. It employs as oracle the following NP set:

$$
E=\left\{\left\langle 0^{n}, C_{1}, C_{2}, y\right\rangle\left|\exists z \in L\left(C_{1}\right) \triangle L\left(C_{2}\right),\right| z \mid \leq n, y \sqsubseteq z\right\}
$$

Note that any other NP-complete set like SAT can be used instead. The learning algorithm is as follows:

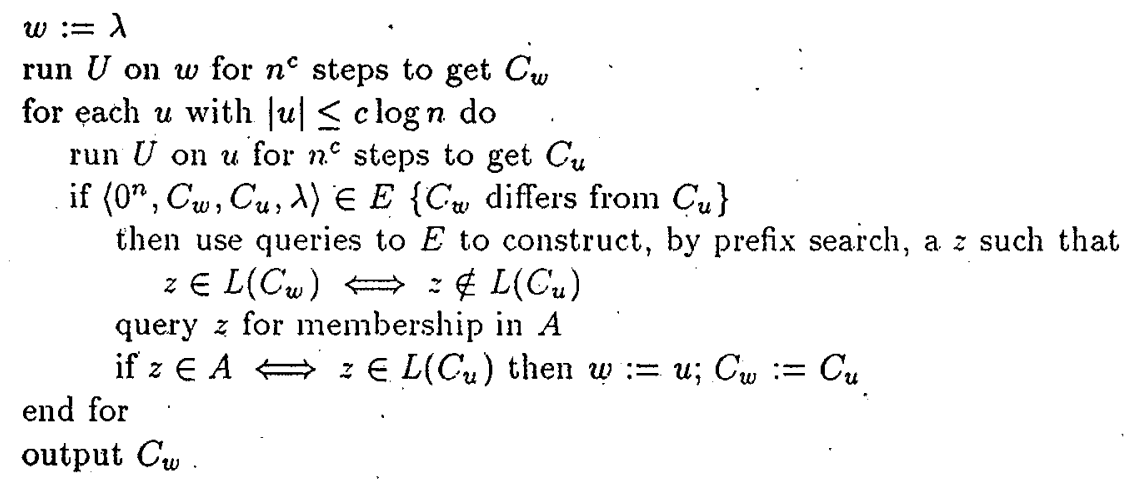

Note that, by hypothesis, one of the $C_{w}$ is correct for $A$; whenever it is considered in the loop, the previously stored values of $w$ and $C_{w}$ are only kept if $E$ is unable to distinguish between them, and otherwise the correct one is kept. So the $\dot{C}_{w}$ reaching the end of the loop must be correct. If appropriate, the seed $w$ can be output instead.

As a consequence, we can prove now the following fact: for $A \in$ Full-P/log, any set in NP relativized to $A$ can be decided in polynomial time with queries to $A$.directly, in the presence of a set in (unrelativized) NP. In the terminology of Structural Complexity, this consequence is a result on "lowness"; more precisely, the sets $A$ for which NPA $\subseteq P^{A \oplus S A T}$ form the first level of the extended low hierarchy, and are denoted as $E L_{1}^{\Sigma}$. Thus the next corollary is actually showing that Full-P/log $\subseteq E L_{1}^{5}$.

Corollary 9. If $A \in$ Full-P/log, then $\mathrm{NP}^{A} \subseteq P^{A \oplus S A T}$.

Proof: This is a standard complexity-theoretic argument. Consider any set $B$ in NP $A$, and assume that polynomial $p(n)$ bounds the length of the queries to $A$ made on inputs of length $n$. Let $x$ be an input of length $n$. Now, use first queries to $A$ and to SAT to run the learner of the previous theorem for target length $p(n)$. Once a circuit expression for $A$, valid up to that length, is obtained, 
a single additional query to SAT with arguments computed from $x$ and that circuit expression indicates whether $x \in B$.

It can be seen that one can do also the reverse: proving directly this corollary and then applying theorem 7 to get the learnability result. The direct proof of corollary 9 is obtained adapting the current proof of theorem 8 to a complexitytheoretic setting. We should also mention that this idea of "playing one circuit expression against another" with the help of an NP oracle is also used in [7], where it is applied to equivalence, queries, and also, implicitly, in an argument about parallel learning in [6]. Corollary 9 also follows, indirectly, from the lowness of instance complexity classes in [2].

There is one more observation to point out: we have now two ways of obtaining a learning algorithm using subset and superset queries. On the one hand, these can simulate equivalence queries in the obvious manner. On the other hand, as shown in [7], when learning a representation class based of boolean models, subset and superset are able to simulate an NP oracle, and of course a membership query. This yields two different, unrelativized, learning algorithms using superset and subset queries.

\section{Learnability from membership queries only}

The purpose of this section is to precisely characterize those concepts for which circuit expressions can be learned in polynomial time using only membership queries, without the additional NP oracle. Note that to construct in polynomial time easy circuit expressions is equivalent to finding their logarithmically long seeds, since these can be found, if necessary, in polynomial time by exhaustive search.

Theorem 10. The following facts are equivalent:

i) $A \in E_{T}$ (Tally2).

ii/ $A$ is decided by a family of easy circuit expressions whose descriptions can be obtained in polynomial time using membership queries.

Proof. First we prove i/ $\Longrightarrow \mathrm{ij} /$. Let $A$ be a set in $E_{T}$ (Tally2), and let $T \in$ Tally2 be such that $T \in \mathrm{P}^{A}$ and $A \in \mathrm{P}^{T}$. Therefore $A \leq n$ can be decided from the characterstic sequence of $T$ up to $n^{c}$ for some $c$. As $T$ is very regular, its characteristic sequence can be expressed relative to the set $\left\{0^{2^{n}} \mid n \geq 0\right\}$ and. then the size of this information is logarithmic in $n$. From this, and the fact that $A \in \mathrm{P}^{T}$, it follows that $A$ has easy circuit expressions as in the characterization given in the previous section. Moreover, these can be constructed from the list of words of $T$ up to length $n^{c}$, and this can be obtained from membership queries to $A$.

Next we see that ii/ $\Longrightarrow \mathrm{i} /$. Let $A$ be a set recognized by a family of easy circuit expressions, and assume that the logarithmically long seeds of the expressions can be obtained in $\mathrm{P}^{A}$. 
We will define a tally set $T$ containing only words of length a power of 2 , and will prove that $A \in P^{T}$. We invoke again the techniques from [5]. The main idea is to keep only the seeds for circuit expressions corresponding to some selected $n$ instead of storing all of them in the oracle $T \subseteq\left\{0^{2^{n}} \mid n \in N\right\}$. Of course, we have to select for the oracle infinitely many of them; but the fact that each circuit expression is good for all the words with length smaller than the $\boldsymbol{n}$ it corresponds to allows us to select them, in principle, arbitrarily far apart.

Now we must find a balance between two contradictory restrictions. If we select seeds for easy circuit expressions to enter the oracle too frequently then they will altogether need too many bits, and some of them will be encoded too far away in the oracle; but if we select them too far apart, then for some words the nearest valid advice would be too long to be extracted from the oracle by a polynomial time machine.

It turns out that there is a way of selecting seeds for which the balance is satisfactory. We will encode in the oracle all the seeds for circuit expressions corresponding to lengths $n=2^{2^{m}}$ for all $m \in N$ and skip all the intermediate ones. Each bit of each selected seed will be encoded as the presence or absence. of a word of the form $0^{2^{m}}$ in the set $T$.

The seed for the easy circuit expression corresponding to length $2^{2^{\mathbf{0}}}$, respectively $2^{2^{2}} \ldots 2^{2^{m}}$, has size $c$, respectively $2 c \ldots 2^{m} c$. We use the first $c$ powers of two, from $0^{2}$ until $0^{2^{c}}$, to encode the seed for length $2^{2^{\circ}}=2$. The second string to be stored has length $2 c$, so this information needs $2 c$ powers of two: use the next ones, from $0^{2^{c+}}$ until $0^{2^{c+3 c}}$. In general, the information of the seed corresponding to the length $2^{2^{m}}$ is encoded in the tally set $T$ by the elements $0^{2^{c+2 c+2^{2}} c+\cdots+2^{m-1} c+1}$ until. $0^{2 c+2 c+2^{2} c+\cdots+2^{m} c}$.

So let $s_{m}$ be the seed of length $c 2^{m}$ from which we produce the circuit expression in $\operatorname{CEX}\left(A, 2^{2^{m}}\right)$, of length (and within time) polynomial in $2^{2^{m}}$, and let $T$ be

$$
T=\left\{0^{2^{\left(\Sigma_{i} \leq m-1\right.}{\left.2^{i} c\right)+p}^{*}} \mid 1 \leq p \leq 2^{m} c \text { such that the } p^{t h} \text { bit of } s_{m} \text { is } 1\right\}
$$

On the one hand we prove that $A \in P^{T}$. On input $x$; find an integer $m$ such that $2^{2^{m-1}}<|x| \leq 2^{2^{m}}$. This can be done in linear time. Observe that this selection ensures that $\log \log |x| \leq m<\log \log \left(|x|^{2}\right)$. Now, for each value of $p$ from 1 to

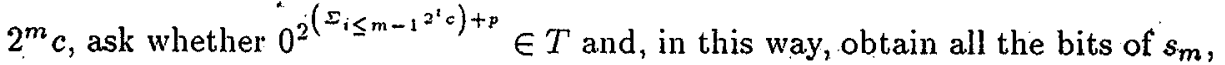
which can now be expanded to a circuit expression which, in turn, allows us to decide in polynomial time whether $x \in A$. It remains to see that the queries can be asked in polynomial time; it suffices to see that they are polynomially long.

The number of queries is bounded by $c \log \left(|x|^{2}\right)$. A bound on the length of the oracle queries is $2^{c+2 c+2^{2} c+\cdots+2^{m} c}=2^{\left(1+2+2^{2}+\cdots+2^{m}\right) c}<2^{2^{m+1} c}$. As $m<$ $\log \log \left(|x|^{2}\right)$, the queries have length at most $2^{c 2^{\log \log \left(|x|^{d}\right)}}=\left(2^{2^{\log \log \left(|x|^{d}\right)}}\right)^{c}=|x|^{d^{\prime}}$ for appropriate constants $d$ and $d^{\prime}$. So $A \in P^{T}$.

On the other hand, $T \in P^{A}$, because to know whether $0^{2^{n}} \in T$, we compute the approprate logarithmically long seed and check whether the bit correspond- 
ing to that element of $T$ is 1 . As $f(n) \in P F^{A}$, then $T \in P^{A}$. So $A$ is Turing equivalent to a tally set $T \in T A L L Y 2$. This implies that $A \in E_{T}(T A L L Y 2)$.

\section{Polylogarithmic complexity}

Of course, it would be interesting to relax the condition of "easy" circuit expressions on which all the constructions are based. Unfortunately, we cannot extend all the results for bounds larger than logarithmic. But, for certain reasonable conditions on the bounds, some parts of the characterization carry through; and, concentrating on polylogarithmic bounds, there are alternative characterizations that extend those given in the last section.

We first observe that a more careful tuning of the proof technique from [5] gives the following result. The essence of the result from there, as extensively used in the previous section, is that from a family of independent logarithmically growing advice words we can find a single infinite sequence such that its prefixes, again of logarithmic length, can be used as advices as well. This is equivalent to the condition that each advice is a prefix of all the following ones. We have been choosing the doubly tally set so that its characteristic function, with respect to $\left\{0^{2^{n}}\right\}$, follows exactly that infinite sequence. Now. our key observation is that, with some additional work, this fact carries over to other advice length bounds.

Theorem 11. Let $f(n)$ be an unbounded function that can be calculated in polynomial time. Then if $A \in$ Full- $\mathrm{P} / O(f(n))$,' it is so via a sequence of advice words in which each is a prefix of all the following ones.

Proof. Let $A \in$ Full-P/O(f(n)), so that there exists a Turing machine $M$, that decides $A$ in polynomial time, with the help of advices $\left\{w_{n}\right\}_{n \in \mathbb{N}}$. There exists a function $h(n) \in O(f(n))$ such that each $w_{n}$ has the length bounded by $h(n)$. Without loss of generality we assume that $h$ is polynomial-time computable and unbounded, too. We have to construct an infinite sequence, in such a way that the prefixes of length $O(f)$ can be used as well as advices for $A$.

Using the fact that any advice corresponding to a particular length can be used by smaller lengths, the infinite sequence could be the concatenation of some $w_{n}$ 's. The idea is again to keep only the information $w_{n}$ for some selected $n$ 's instead of storing all of them. We have to consider again a similar balance between selecting $w_{n}$ 's not too frequently, to avoid the use of too many bits, and selecting them too separated, lest for some words the nearest valid advice be too long.

Since $h$ is unbounded, it is possible to find for each $n$ a number $m_{n}$ such that $2^{m_{n}-1}<h(n) \leq 2^{m_{n}}$; moreover, given $n$, the search for $m_{n}$ can be done in polynomial time. Remark that eventually many $n$ 's are associated to the same $m_{n}$. Let $g(n)$ be the following function:

$$
g(n)=\max \left\{i \mid h(i) \leq 2^{m_{n}}\right\}
$$


Construct an infinite sequence $\beta$ including all of $\left\{w_{g(n)}\right\}_{n \in N}$. In order to get a good manipulation of the sequence, we construct $\beta$ step by step, appending a power of two many bits each time. Namely $\beta=p_{1} p_{2} p_{3} \ldots p_{n} \ldots$ in such a way that for all nonzero $i$ it holds:

$$
p_{i}= \begin{cases}\underbrace{w_{g(n)} 10 \ldots 0}_{2^{i}} & \text { if } 2^{i}=2^{m_{n}} \text { for some } n \\ \underbrace{0 \ldots 0^{2}}_{2^{m_{n}}} & \text { otherwise }\end{cases}
$$

The minimum prefix of $\beta$ containing the information of the advice $w_{g(n)}$ will be exactly $\beta_{1: 2^{m} n^{+1}-1}$, which denotes the finite word made up with the bits of $\beta$ starting at 1 and ending at $2^{m_{n}+1}-1$. The following algorithm decides whether a word $x$ is in $A$, using $O(f)$ initial bits of $\beta$ as advice:

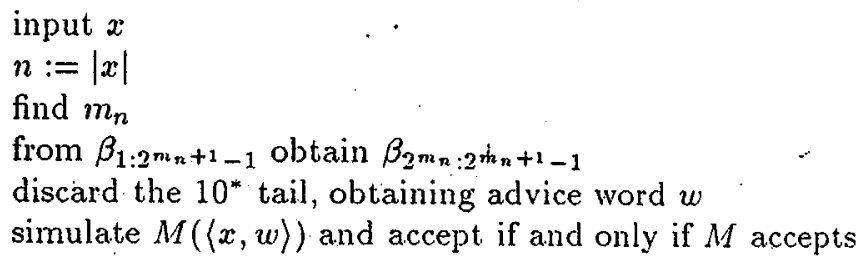

The advice length is exactly $2^{m_{n}+1}-1$. From the fact that $2^{m_{n}-1}<h(n)$, we obtain that $m_{n}<\log (h(n))+1$, so $2^{m_{n}+1}-1$ is bounded by $4 h(n) \in O(f(n))$.

A similar construction appears in [4], the essential difference being that in their context' the time bounds for the Kolmogorov complexity definitions are based on the length of the output, and this requires a number of additional hypothesis and extra information manipulation.

We will focus next in the nonuniform classes defined from polylog advices, that is, Full-P/ $O\left(\log ^{i}(n)\right)$. The next question we address is how to extend the characterization of the sets with easy circuit expressions learnable only from membership queries, to not-so-easy (e.g. polylog-easy) circuit expressions. Observe that nowhere in the class $E_{T}$ (Tally2) appears explicitly a logarithm that could be changed into a polylog function. Actually, as it turns out, there is a nice reason for this.

Indeed, as we shall see shortly, there is a marked difference between the logarithmic and the polylogarithmic case. Both allow for a characterization in terms of polynomial-time. Turing degrees of tally sets. In the case discussed in the previous section, the tally sets used were defined by a "qualitative" condition that the words were only of certain fixed lengths, namely powers of 2 . When we move to polylog functions, the characterization no longer has this flavor, but it instead becomes purely "quantitative": it corresponds to tally degrees of polylog density. Moreover, the exponent of the density tightly corresponds to the exponent of the complexity of the circuit expressions, modulo a log factor; and this log factor precludes the use of this characterization for the logarithmic case. So the theorem of the previous section cannot be obtained as a particularization of the present one. 
Let us denote as $\mathcal{F}$-Tally the class of tally sets of density bounded by a function from $\mathcal{F}$, i.e., having at most $f(n)$ words up to length $n$ where $f \in \mathcal{F}$.

Lemma 12. For all $i \geq 1$, the following holds:

$$
\mathrm{P}_{T}\left(O\left(\log ^{i}(n)\right)-\text { Tally }\right)=\text { Full-P } / O\left(\log ^{i+1}(n)\right)
$$

Proof. We show first $\mathrm{P}_{T}\left(O\left(\log ^{i}(n)\right)\right.$-Tally $) \subseteq$ Full-P $/ O\left(\log ^{i+1}(n)\right)$. In polynomial time, at most a polynomially long initial part of the tally set is accessible, and we need to know the tally oracle $T$ up to that length. This information will fit into $O\left(\log ^{i+1}(|x|)\right)$ bits, and will constitute our advice word. More precisely, suppose that $A \in \operatorname{P}_{T}\left(O\left(\log ^{i}(n)\right)\right.$-Tally $)$. That means there is a deterministic Turing machine $M$ that decides $A$ querying a tally set $T$ of $d \log ^{i}(n)$ density, and working in polynomial timie. Let $n^{c}$ be the bound in the number of the steps that the machine $M$ can perform. If we want to see whether an input $x$ belongs to the set $A$, then we need to know $T$ up to length $|x|^{c}$. This information is encoded in $O(\log (|x|)) * O\left(\log ^{i}\left(|x|^{c}\right)\right)=O\left(\log ^{i+1}(|x|)\right)$ bits, and it can be used as advice in order to decide $A$. Moreover, with this advice, we can decide all the words of length less or equal to $|x|$. Therefore, $A \in$ Full-P $/ O\left(\log ^{i+1}(n)\right)$.

Conversely, we see that Full-P $/ O\left(\log ^{i+1}(n)\right) \subseteq \mathrm{P}_{T}\left(O\left(\log ^{i}(n)\right)\right.$-Tally $)$. By the previous theorem, we can assume that there is a single infinite sequence limiting the successive advices. We encode it into a tally set of the indicated density.

The encoding scheme is as follows. We construct a tally set $T$ having at most $r n^{i-1}$ words in the interval between $0^{2^{n}}$ and $0^{2^{n+1}}$, for some constant $r$. These must be.chosen among the $2^{n}$ words of the interval. So, we divide the $2^{n}$ words into $r n^{i-1}$ parts, and select for $T$ at most one word per part. Each part has (at least) the following number of words to choose from:

$$
\mathcal{N}=\left\lfloor\frac{2^{n}}{r n^{i-1}}\right\rfloor
$$

So, we can interpret each part as a digit in base $\mathcal{B}=\mathcal{N}+1$, depending on where the only word (if at all) of that part is, and in this way encode $\log \mathcal{N}=$ $n-\log r-(i-1) \log n$ bits per part. The total length encoded can be therefore up to $r n^{i}-r n^{i-1} \log r-r n^{i-1}(i-1) \log n$ bits.

This is enough room to encode the needed part of the infinite sequence limiting the advices. Indeed, assuming that we have managed to encode up to the word $0^{2^{n}}$ the first $c \log ^{i}\left(2^{n}\right)=c n^{i}$ bits of the infinite sequence, we must now. encode additional ones up to $c \log ^{i}\left(2^{n+1}\right)=c(n+1)^{i}=c n^{i}+O\left(n^{i-1}\right)$ bits; this means that only $O\left(n^{i-1}\right)$ bits are new, and they fit in the available space if $r$ is chosen appropriately.

The total density of the tally set is $\sum_{n=0}^{m-1} r n^{i-1}$ up to length $M=2^{m}$, which sums less than $r m^{i}=r \log ^{i} M$. So the density is as required. The advice can be easily decoded in polynomial time, since it is just essentially changing the representation of a number into base 2 .

Finally, through a combination of all our proof methods we can also obtain: 
Theorem 13. For all $i \geq 1$, the class of sets having polynomial size circuit expressions in $\mathrm{K}\left[O\left(\log ^{i+1}(n)\right)\right.$, poly] whose descriptions are learnable in polynomial time from membership queries is precisely $E_{T}\left(O\left(\log ^{i}(n)\right)\right.$-Tally).

Proof (Sketch). Use the encoding described in the lemma to code into a tally set the seeds for the circuit expressions found by the learning algorithm. Not all of them are to be encoded: those are to be selected as in the proof of theorem 11. This yields a tally set that is T-equivalent to the learned concept. The converse follows exactly as in the proof of the main result of the previous section.

Taking the union over all $i$ gives:

Corollary 14. The class of sets that have a polynomial size circuit expression in $\mathrm{K}\left[O\left(\log ^{O(1)}(n)\right)\right.$, poly], and whose description is learnable in polynomial time from membership queries, is precisely $E_{T}\left(O\left(\log ^{O(1)}(n)\right)\right.$-Tally).

This corollary proves that to learn all of $P_{T}\left(O\left(\log ^{O(1)}(n)\right)\right.$-Tally) would require either more than polynomial time, or more complex queries, or additional oracles. We have not found a nontrivial learning algorithm of any of these sorts for them, and leave this question as open.

\section{References}

1. D. Angluin: "Queries and Concept Learning". Machine Learning 2 1988, 319-342.

2. V. Arvind, J. Köbler, M. Mundhenk: "Lowness and the complexity of sparse and tally descriptions". In: Third Annual Symposium on Algorithms and Computation, 1992.

3. J. L. Balcázar, J. Díaz, J. Gabarró: Structural Complexity I. Springer Verlag 1988.

4. J. L. Balcázar, R: Gavaldà, M. Hermo: "On infinite sequences almost as easy as $\pi$ ". Workshop on Applications of Descriptional Complexity, Rutgers Univ., july 1994. Report LSI-94-24-R, Univ. Politècnica de Catalunya.

5. J. L. Balcázar, M. Hermo, E. Mayordomo: "Characterizations of logarithmic advice complexity classes". In: Algorithms, Software, Architectüre: Information Processing 92, Elsevier 1992, vol. 1, 315-321.

6. N. Bshouty, R. Cleve: "Pn the exact learning of formulas in parallel". In: IEEE Symp. Foundations of Computer: Science, IEEE Computer Society Press 1992, 513522.

7. N. Bshouty, R. Cleve, S: Kamnan, C. Tamon: "Oracles and queries that are sufficient for exact learning". In: Conference on Computational Learning Theory 1994, 130139.

8. R. Gavaldà: "The complexity of learning with queries". In: Structure in Commplexity Theory 1994, 324-337.

9. M. Hermo: "Degrees and reducibilities of easy tally sets". In: Mathematical Foundations of Computer Science 94, Springer-Verlag Lecture Notes in Computer Science 8411994, 403-412.

10. J. Köbler: "Locating $\mathrm{P} /$ poly optimally in the low hierarchy". In: Symp. Theoretical Aspects of Computer Science 93, Springer-Verlag Lecture Notes in Computer Science 665 1993, 28-37. 
11. M. Li, P. Vitányi: "Learning simple concepts under simple distributions". SIAM Journal on Computing 20 1991, 911-935.

12. M. Li, P. Vitányi: An introduction to Kolmogorov complexity and its applications. Springer-Verlag 1994.

13. O. Watanabe: "A framework for polynomial time query learnability". Mathematical Systems Theory 27 1994, 211-229.

14. O. Watanabe, R. Gavaldà: "Structural analysis of polynomial time query learnability". Mathematical Systems Theory 27 1994, 231-256. 


\section{Departament de Llenguatges i Sistemes Informàtics \\ Universitat Politècnica de Catalunya}

Research Reports - 1995

LSI-95-1-R "Octree simplification of polyhedral solids", Dolors Ayala and Pere Brunet.

LSI-95-2-R "A note on learning decision lists", Jorge Castro.

LSI-95-3-R "The complexity of searching implicit graph", José L. Balcázar.

LSI-95-4-R "Design quality metrics for object-oriented software development", Alonso Peralta, Joan Serras; and Olga. Slavkova.

LSI-95-5-R "Extension orderings", Albert Rubio.

LSI-95-6-R "Triangles, ruler, and compass"; R. Juan-Arinyo.

LSI-95-7-R "The modifiability factor in the LESD project: definition and practical results", Nuria Castell and Olga Slavkova.

LSI-95-8-R "Learnability of Kolmogorov-easy circuit expressions via queriès", José L. Balcázar, Harry Buhrman, and Montserrat Hermo.

Copies of reports can be ordered from:

Nuria Sánchez

Departament de Llenguatges i Sistemes Informàtics

Universitat Politècnica de Catalunya

Pau Gargallo, 5

. 08028 Barcelona, Spain

secrelsiolsi.upc:es 